\title{
Extensión de la afectación pulmonar por tomografía en pacientes con neumonía por SARS-CoV-2
}

\section{Extent of lung involvement by tomography in patients with SARS-CoV-2 pneumonia}

\author{
Jaime A. Collins ${ }^{1, a}$, Rafael P. Ramos ${ }^{2, b}$, Fiorella V. Loyola ${ }^{1, c}$, Iván A. Meza ${ }^{2, d}$, Gerson E. Díaz $^{3, e}$, Iván P. Márquez ${ }^{2, f}$ \\ ${ }^{1}$ Servicio de Infectología, Departamento de Medicina Interna, Hospital Nacional Guillermo Almenara Irigoyen, EsSalud. Lima, Perú. \\ ${ }^{2}$ Servicio de Ecografia y Tomografía, Departamento de Diagnóstico por Imágenes, Hospital Nacional Guillermo Almenara Irigoyen, EsSalud. Lima, Perú. \\ ${ }^{3}$ Departamento de Emergencia, Hospital Nacional Guillermo Almenara Irigoyen, EsSalud. Lima, Perú. \\ ${ }^{a}$ Médico especialista en medicina interna y en enfermedades infecciosas; doctor en gobierno y politica pública. ORCID: https://orcid.org/0000-0002-4168-3157 \\ ${ }^{\mathrm{b}}$ Médico especialista en radiología, ORCID: https://orcid.org/0000-0002-3160-1437 \\ ${ }^{c}$ Médica residente de medicina interna, ORCID: https://orcid.org/0000-0002-2224-4095 \\ ${ }^{\mathrm{a}}$ Médico especialista en radiología, ORCID: https://orcid.org/0000-0002-50221-3064 \\ ${ }^{e}$ Médico especialista en medicina de emergencias y desastres, ORCID: https://orcid.org/0000-0002-3046-5816 \\ ${ }^{\dagger}$ Médico especialista en radiologia, ORCID: https://orcid.org/0000-0001-6356-6026
}

\section{An Fac med. 2021;82(2):113-7. / DOI: https://doi.org/10.15381/anales.v82i2.19707}

\section{Correspondencia: \\ Jaime Antonio Collins Camones \\ jcollins/p@gmail.com}

Recibido: 16 de febrero 2021

Aprobado: 14 de junio 2021

Publicación en línea: 23 de julio 2021

Conflictos de interés: Los autores declaran no tener conflictos de interés.

Fuente de financiamiento: Autofinanciado

Citar como: Collins J, Ramos R, Loyola F, Meza I, Díaz G, Márquez I. Extensión de la afectación pulmonar por tomografía en pacientes con neumonía por SARSCoV-2. An Fac med. 2021;82(2):113-7. DOI: https:/doi.org/10.15381/anales. v82i2.19707

\section{Resumen}

Objetivo. Determinar la extensión de la afectación pulmonar en pacientes con neumonía por SARS-CoV-2 mediante tomografía. Método. Evaluación retrospectiva en pacientes con evidencia de COVID-19 del Hospital Nacional Guillermo Almenara Irigoyen, Lima - Perú, al inicio de la pandemia COVID-19, entre el 15 de marzo y el 14 de mayo de 2020. La extensión de la neumonía se determinó mediante tomografía con base en la Clasificación de la Sociedad Francesa de Imagen Torácica. Resultados. Se incluyeron en el estudio 485 pacientes. La extensión de la neumonía fue: ausente 1,2\%, mínima 4,9\%, moderada 20,6\%, extensa $27,4 \%$, grave $30,7 \%$ y crítica $15,1 \%$. La afectación pulmonar se asoció con edad mayor de 60 años $(p=0,014)$ y saturación de oxígeno ambiental por debajo de $90 \%(n=372, p=0,000)$. Conclusiones. Por su extensión, las neumonías por SARS-CoV-2 en los primeros dos meses de la epidemia de COVID-19 en el Hospital Almenara fueron graves, extensas y moderadas en su gran mayoría. La extensión de la neumonía se asoció con edad y saturación de oxígeno ambiental al ingreso.

Palabras clave: COVID-19; Neumonía; Tomografía; Índice de Gravedad de la Enfermedad; Perú (Fuente: DeCS BIREME).

Abstract

Objective. To determine the extent of pulmonary involvement in patients with SARS-CoV-2 pneumonia using tomography. Method. Retrospective evaluation in patients with evidence of COVID-19 at the Guillermo Almenara Irigoyen National Hospital, Lima - Peru, at the beginning of the COVID-19 pandemic, between March 15 and May 14, 2020. The extent of pneumonia was determined by means of tomography based on the Classification of the French Society of Thoracic Imaging. Results. 485 patients were included in the study. The extent of pneumonia was: $1.2 \%$ absent, $4.9 \%$ minimal, $20.6 \%$ moderate, $27.4 \%$ extensive, $30.7 \%$ severe, and $15.1 \%$ critical. Lung involvement was associated with age older than 60 years $(p=0.014)$ and ambient oxygen saturation below $90 \%(n=372, p=0.000)$. Conclusions. Due to its extension, the SARSCoV-2 pneumonia in the first two months of the COVID-19 epidemic at Hospital Almenara were severe, extensive and mostly moderate. The extent of pneumonia was associated with age and ambient oxygen saturation at admission.

Keywords: COVID-19; Pneumonia; Tomography; Severity of IIIness Index; Peru (source: MeSH NLM). 


\section{INTRODUCCION}

La pandemia de la COVID-19 sigue vigente en el mundo con varios epicentros. Más de cien millones de casos confirmados y más de dos millones de muertos ${ }^{(1)}$. Las imágenes del tórax son una herramienta de apoyo diagnóstico importante, sobre todo en un estadio temprano de la enfermedad, cuando las pruebas diagnósticas de laboratorio son negativas o no están disponibles ${ }^{(2)}$. Desde los inicios de la pandemia, varios estudios por imágenes han caracterizado a la neumonía por SARS-CoV-2 tanto en cohortes pequeñas ${ }^{(3-6)}$ como grandes ${ }^{(7,8)}$, incluyendo revisiones sistemáticas ${ }^{(9,10)}$. No obstante, existe limitada información sobre la determinación de la extensión de esta neumonía mediante imágenes en general y tomografía en particular. Con base en ello, el presente estudio se propuso como objetivo determinar la extensión de la neumonía por SARSCoV-2 mediante tomografía en pacientes hospitalizados, y evaluar su asociación con edad, sexo y el nivel de saturación de oxígeno ambiental al ingreso de los pacientes a la sala de emergencia.

\section{MÉTODOS}

Se realizó un estudio observacional, retrospectivo y de corte transversal.

\section{Población de estudio}

Estuvo conformada por todos los pacientes con evidencia etiológica de COVID-19 quienes fueron admitidos en el Departamento de Emergencia del Hospital Nacional Guillermo Almenara Irigoyen, EsSalud, Lima - Perú, entre el 15 de marzo y el 14 de mayo de 2020.

\section{Revisión radiológica}

Para el estudio, dos radiólogos (RPR, IAM) revisaron las imágenes digitales de las tomografías de tórax sin contraste tomadas al ingreso de los pacientes al hospital. Cuando hubo discrepancia, un tercer radiólogo (IPM) fue dirimente. El índice de dirimencia fue de 2,7\% (13/485). La extensión de la neumonía se determinó mediante una adaptación de la Propuesta de Reporte Estructurado para casos de COVID-19 de la Sociedad Francesa de Imagen Torácica ${ }^{(11)}$. En tal sentido, la estimación de la extensión se realizó mediante evaluación visual convencional de las tomografías de tórax sin contraste, sin uso de software de volumetría pulmonar, ni inteligencia artificial. Cada uno de los cinco lóbulos pulmonares -dos superiores, dos inferiores y el lóbulo medio- fueron evaluados con imágenes axiales y reformación tanto coronal como sagital. Tomando en cuenta el porcentaje de compromiso lobar se utilizó la siguiente puntuación ${ }^{(12)}$ : sin compromiso $=0$ puntos, $1-5 \%=1$ punto, $6-25 \%=2$ puntos, $26-50 \%$ $=3$ puntos, $51-75 \%=4$ puntos, $>75 \%=5$ puntos, tal como se muestra se muestra en la figura 1.

De este modo, la estimación de la extensión pulmonar global de la neumonía por SARS-CoV-2, según porcentaje de afectación, se estableció en las siguientes categorías de la clasificación francesa: ausente (0\%), mínima (1 a 9\%), moderada (10 a $24 \%$ ), extensa (25 a $49 \%$ ), grave (50 a $74 \%$ ) o crítica (mayor o igual a 75\%), tal como se muestra en la figura 2.

\section{Análisis estadístico}

El análisis se hizo con estadística descriptiva, y la prueba de Ji-cuadrado o prueba exacta de Fisher para determinar si la extensión de la afectación pulmonar estuvo asociada a la edad, sexo y saturación de oxígeno ambiental al ingreso de los pacientes.

\section{Aspectos éticos}

Se revisaron fuentes secundarias de datos. Se preservó la confidencialidad de los datos.

\section{RESULTADOS}

Se incluyeron en el estudio un total de 485 casos de COVID-19 confirmados: $57 \%(277 / 485)$ mediante RT-PCR y el resto 43\% (208/485) mediante prueba rápida de anticuerpos. Se excluyeron 48 casos confirmados de COVID-19 por no contar con tomografía o no estar disponible en el sistema digital de imágenes médicas del hospital. La media de edad de la cohorte entera de pacientes fue de 62 años con una desviación estándar de 15 años. Y el porcentaje de mujeres fue $29 \%$ (141/485). La distribución de las ca-

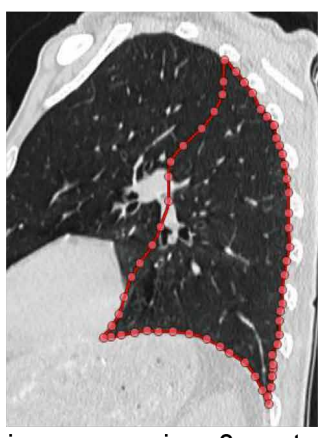

sin compromiso $=0$ puntos

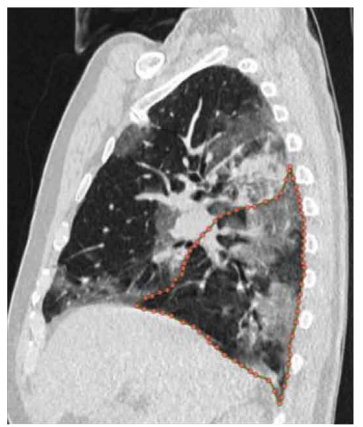

$26-50 \%=3$ puntos

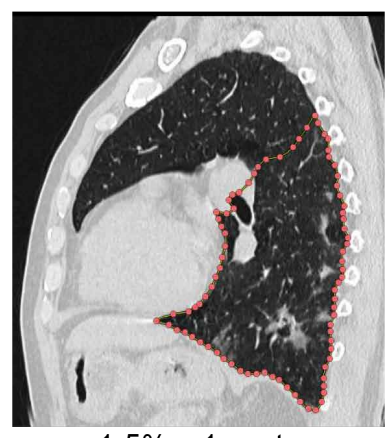

$1-5 \%=1$ punto

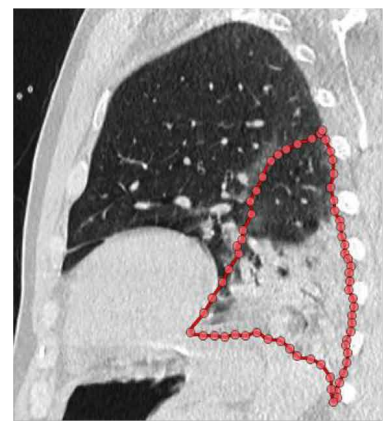

$51-75 \%=4$ puntos

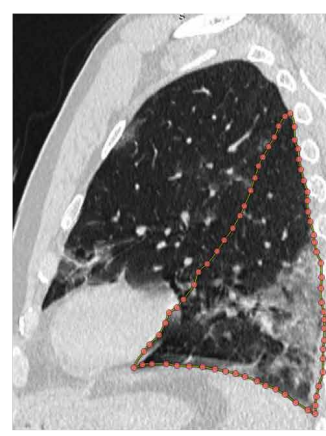

$6-25 \%=2$ puntos

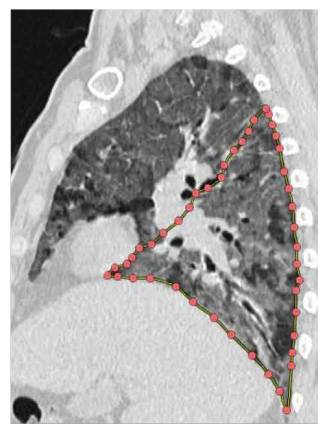

$>75 \%=5$ puntos

Figura 1. Grado de compromiso lobar según la adaptación de la Propuesta de Reporte Estructurado para casos de COVID-19 de la Sociedad Francesa de Imagen Torácica

Nota: Estimado como el procentaje de afección pulmonar tras evaluación visual según los rangos mostrados. En esta imagen se ha resaltado el lóbulo inferior derecho para mejor visualización. Elaboración propia con imágenes en reformación sagital de seis pacientes de nuestro estudio. 
A

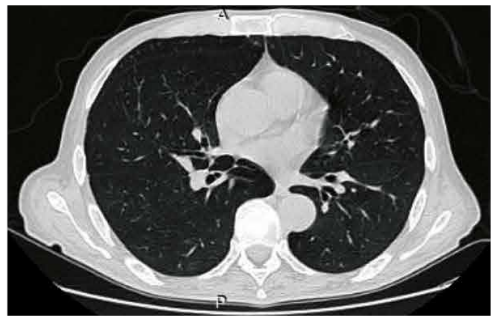

B

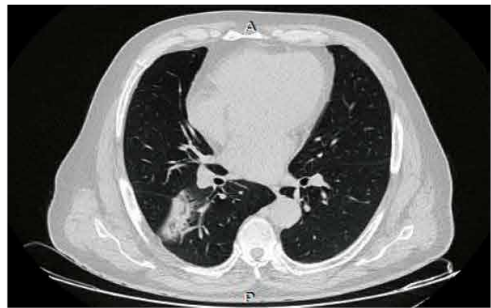

C

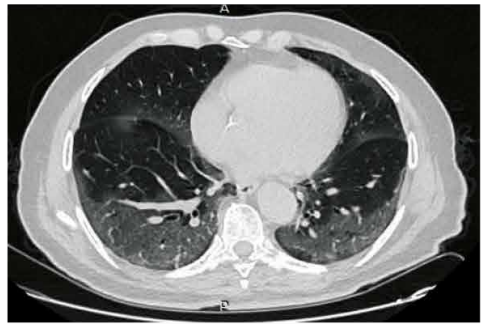

Extensión: mínima (1-9\%)

D

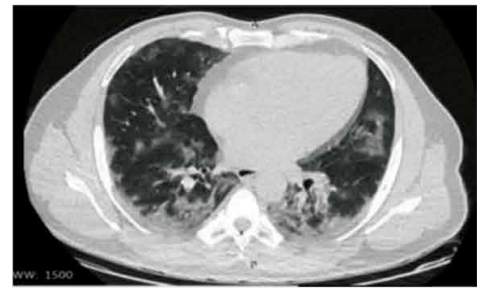

Extensión: moderada (10-24\%)
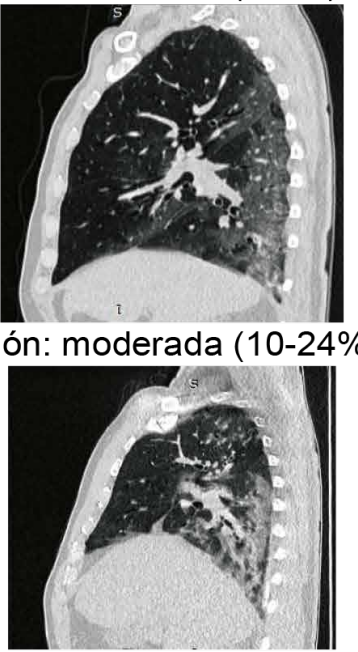

Extensión: extensa (25-49\%)

E
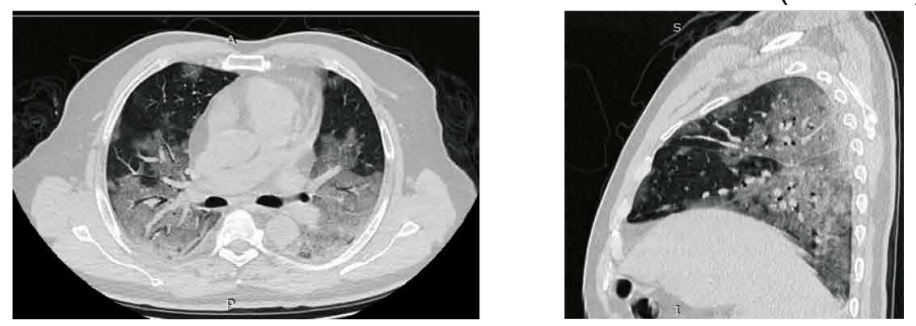

Extensión: grave (50-74\%)

$\mathbf{F}$
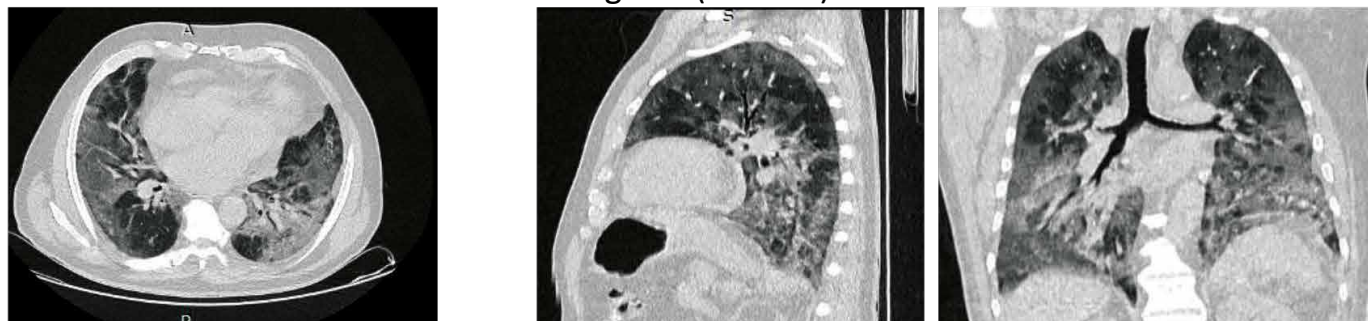

Extensión: crítica ( $\geq 75 \%)$

Figura 2. Extensión pulmonar global de la neumonía por SARS-CoV-2 según la adaptación de la Propuesta de Reporte Estructurado para casos de COVID-19 de la Sociedad Francesa de Imagen Torácica.

Nota: Se muestran los grados de compromiso pulmonar global en rangos de de porcentaje de afectación pulmonar teniendo en cuenta el volumen pulmonar bilateral. Elaboración propia con casos de nuestro estudio. 
Tabla 1. Extensión de la neumonía según edad en pacientes con neumonía por SARS-CoV-2. Hospital Nacional Guillermo Almenara, EsSalud, marzo - mayo 2020

\begin{tabular}{lccc} 
Extensión & Total & Mayores de $\mathbf{6 0}$ años & $\mathbf{6 0}$ años o menos \\
& $\mathbf{N}=\mathbf{4 8 5}$ & $\mathbf{N}=\mathbf{2 7 0}$ & $\mathbf{N}=\mathbf{2 1 5}$ \\
\hline Ausente o mínima & $30(6,2 \%)$ & $12(4,4 \%)$ & $18(8,4 \%)$ \\
\hline Moderada & $100(20,6 \%)$ & $46(17 \%)$ & $54(25,1 \%)$ \\
\hline Extensa & $133(27,4 \%)$ & $72(26,7 \%)$ & $61(28,4 \%)$ \\
\hline Grave & $149(30,7 \%)$ & $91(33,7 \%)$ & $58(26,9 \%)$ \\
\hline Crítica & $73(15,1 \%)$ & $49(18,2 \%)$ & $24(11,2 \%)$ \\
\hline
\end{tabular}

Prueba exacta de Fisher, $p=0,014$

tegorías de extensión de la neumonía por SARS-CoV-2 fue la siguiente: ausente 6 (1,2\%), mínima 24 (4,9\%), moderada 100 $(20,6 \%)$, extensa $133(27,4 \%)$, grave 149 $(30,7 \%)$ y crítica $73(15,1 \%)$.

Hubo asociación entre la extensión de la neumonía con la edad: las proporciones de neumonías graves y críticas fueron mayores en pacientes con más de 60 años de edad que en pacientes con 60 años o menos: $34 \%$ versus $27 \%$ y $18 \%$ versus $11 \%$, respectivamente, $p=0,014$ (Tabla 1). No hubo asociación con el sexo $(p=0,191)$. Por otra parte, también hubo asociación con el nivel de saturación ambiental de oxígeno al ingreso al hospital: las proporciones de neumonías graves y críticas fueron mayores en pacientes con saturación por debajo de $90 \%$ que en pacientes con saturación por encima o igual a $90 \%$ : $43 \%$ versus $15 \%$ y $24 \%$ versus $4 \%$, respectivamente, $p=0,000$ (Tabla 2 ).

\section{DISCUSIÓN}

El estudio propuesto presenta la evaluación más grande a nivel nacional y una de las más grandes a nivel internacional de la neumonía por SARS-CoV-2 mediante tomografía en pacientes hospitalizados en un establecimiento de salud con nivel de atención III-2 ${ }^{(13)}$. El foco de atención del estudio fue la extensión de la neumonía al momento del ingreso de los pacientes al hospital. En su gran mayoría los pacientes presentaron neumonías graves, extensas y moderadas. Del mismo modo, los pacientes adultos mayores así como los pacientes con saturación de oxígeno ambiental por debajo de $90 \%$ al ingreso presentaron significativamente mayores proporciones de neumonías graves y críticas.

La neumonía por SARS-CoV-2 ha sido caracterizada mediante tomografía tanto en estudios transversales ${ }^{(7)}$ como longitudinales ${ }^{(14,15,16)}$. Incluso en este último tipo de estudios se ha llegado a identificar o plantear cuatro $^{(12,17)}$ o cinco fases evolutivas ${ }^{(18)}$ de la neumonía por imágenes. Adicionalmente, la extensión de la neumonía puede tener un correlato con la gravedad clínica de los pacientes ${ }^{(8)}$, con la progresión ${ }^{(19)}$ y con el desenlace de los mismos ${ }^{(20)}$. En este sentido, la clasificación utilizada en el presen- te estudio resulta de especial interés para los clínicos, sobre todo frente a pacientes hospitalizados y en centros como el nuestro donde la mayor parte de la población admitida es adulta mayor con una carga importante de enfermedades preexistentes.

Por otra parte, las limitaciones de disponibilidad y precisión diagnóstica de las pruebas de laboratorio para COVID-19 han hecho que el recurso de la tomografía se emplee de forma considerable, lo que no solo ha ocurrido en nuestro medio ${ }^{(21,22)}$. No obstante, su uso no debe ser generalizado ${ }^{(23)}$. La tomografía debería restringirse a los pacientes con sospecha o diagnóstico confirmado de COVID-19 con indicación de hospitalización en función de la saturación de oxígeno ambiental $(<95 \%)$ y factores de riesgo. La estimación de la extensión podría hacerse de manera práctica en una presentación sagital, tomando en cuenta la división pulmonar a través de las cisuras oblicuas o mayor, ya que está presente en ambos pulmones, como un límite para estimar la afección en aproximadamente un 50\% del volumen total pulmonar.

Tabla 2. Extensión de la neumonía según saturación de oxígeno ambiental en pacientes con neumonía por SARS-CoV-2. Hospital Nacional Guillermo Almenara, EsSalud, marzo - mayo 2020.

\begin{tabular}{lccc} 
Extensión & Total & Saturación $<\mathbf{9 0 \%}$ & \multicolumn{1}{c}{ Saturación $>=\mathbf{9 0 \%}$} \\
& $\mathbf{N}=\mathbf{3 7 2}$ & $\mathbf{N}=\mathbf{1 8 1}$ & $\mathbf{N}=\mathbf{1 9 1}$ \\
\hline Ausente o mínima & $24(6,5 \%)$ & $\mathbf{1}(0,6 \%)$ & $23(12 \%)$ \\
\hline Moderada & $86(23,1 \%)$ & $19(10,5 \%)$ & $67(35,1 \%)$ \\
\hline Extensa & $106(28,5 \%)$ & $40(22,1 \%)$ & $66(34,6 \%)$ \\
\hline Grave & $106(28,5 \%)$ & $78(43,1 \%)$ & $28(14,7 \%)$ \\
\hline Crítica & $50(13,4 \%)$ & $43(23,7 \%)$ & $7(3,6 \%)$ \\
\hline
\end{tabular}

Prueba exacta de Fisher, $p=0,000$ 
En el presente estudio la gran mayoría de los pacientes presentaron al ingreso neumonías graves, extensas y moderadas. No obstante, la situación fue peor en pacientes adultos mayores quienes presentaron más neumonías graves y críticas al momento de la admisión al hospital. Este comportamiento no es extraño si se toman en cuenta reportes de pacientes de mayor edad con mayores proporciones de lesiones consolidadas o atípicas $(8,10)$ que los pacientes de menor edad. Una situación similar ocurrió en quienes presentaron una saturación de oxigeno ambiental por debajo de $90 \%$ al ingreso, lo que muestra una relación entre ambas variables, como era de esperar.

Dos limitaciones importantes del estudio deben ser señaladas. Primero, el ser transversal. La evaluación fue restringida solo al momento de la admisión al hospital. Segundo, el ser descriptivo. Fuera de la edad, sexo y el nivel de saturación de oxígeno ambiental, la extensión de la enfermedad no se ha analizado con otras variables como el desenlace clínico de los pacientes. En contraposición a ello, el tamaño de la población estudiada es grande y la revisión de las imágenes fue planeada y llevada a cabo de forma sistemática.

En conclusión, la extensión de la afectación pulmonar por tomografía en pacientes con neumonía por SARS-Cov-2 admitidos en el Hospital Almenara en los primeros dos meses de la epidemia en nuestro medio fue grave, extensa y moderada en su gran mayoría. Asimismo, los pacientes adultos mayores y quienes tuvieron una saturación de oxígeno ambiental por debajo de $90 \%$ al ingreso tuvieron significativamente mayores proporciones de neumonías graves y críticas.

\section{REFERENCIAS BIBLIOGRÁFICAS}

1. WHO Coronavirus Disease (COVID-19) Dashboard [Internet]. [Fecha de acceso: 13 de febrero
2021]. Disponible en: https://covid19.who.int/table

2. Ai T, Yang Z, Hou H, Zhan C, Chen C, Lv W, et al. Correlation of Chest CT and RT-PCR Testing for Coronavirus Disease 2019 (COVID-19) in China: A Report of 1014 Cases. Radiology. 2020 Aug; 296(2):E32-E40. DOI: 10.1148/radiol.2020 200642

3. Shi H, Han X, Jiang N, Cao Y, Alwalid O, Gu J, et al. Radiological findings from 81 patients with $\mathrm{CO}$ VID-19 pneumonia in Wuhan, China: a descriptive study. Lancet Infect Dis. 2020 Apr; 20(4):425-434. DOI: 10.1016/S1473-3099(20)30086-4

4. Trelles M, Guzmán O, Daly A, Pichilingue J, Luchesi E. Tomografia de tórax y RT-PCR en casos sospechosos de COVID-19 vistos en el servicio de emergencia de Clínica Delgado. Rev Per Radiol. 2020; 20:48-54.

5. Wong HYF, Lam HYS, Fong AH, Leung ST, Chin TW, Lo CSY, et al. Frequency and Distribution of Chest Radiographic Findings in Patients Positive for COVID-19. Radiology. 2020 Aug; 296(2):E72-E78 DOI: 10.1148/radiol.2020201160

6. Carvalho A, Cunha R, Lima BA, Pereira JM, Madureira AJ. Chest CT imaging features of COVID-19 pneumonia: First radiological insights from Porto, Portugal. Eur J Radiol Open. 2020; 7:100294. DOI: 10.1016/j.ejro.2020.100294

7. Guan WJ, Ni ZY, Hu Y, Liang WH, Ou CQ, He JX, et al. Clinical Characteristics of Coronavirus Disease 2019 in China. N Engl J Med. 2020 Apr 30; 382(18): 1708-1720. DOI: 10.1056/NEJMoa2002032

8. Feng Y, Ling Y, Bai T, Xie Y, Huang J, Li J, Xiong $W$, et al. COVID-19 with Different Severities: A Multicenter Study of Clinical Features. Am J Respir Crit Care Med. 2020 Jun 1; 201(11): 1380-1388. DOI: 10.1164/rccm.202002-0445OC

9. Bao C, Liu X, Zhang H, Li Y, Liu J. Coronavirus Disease 2019 (COVID-19) CT Findings: A Systematic Review and Meta-analysis. J Am Coll Radiol. 2020 Jun; 17(6):701-709. DOI: 10.1016/j. jacr.2020.03.006

10. Salehi S, Abedi A, Balakrishnan S, Gholamrezanezhad A. Coronavirus Disease 2019 (COVID-19): A Systematic Review of Imaging Findings in $919 \mathrm{~Pa}-$ tients. AJR Am J Roentgenol. 2020 Jul; 215(1):8793. DOI: 10.2214/AJR.20.23034

11. La société d'Imagerie Thoracique propose un compte-rendu structuré de scanner thoracique pour les patients suspects de COVID-19. SFR Bulletin 25 marzo 2020 - TDM thoracique [Internet]. [Fecha de acceso: 13 de febrero 2021]. Disponible en: https://ebulletin.radiologie.fr/actualitescovid-19/societe-dimagerie-thoracique-proposecompte-rendu-structure-scanner-thoracique

12. Pan $F$, Ye $T$, Sun $P$, Gui $S$, Liang $B$, Li L, et al. Time Course of Lung Changes at Chest CT during Recovery from Coronavirus Disease 2019 (COVID-19). Radiology. 2020 Jun; 295(3):715-721. DOI: $10.1148 /$ radiol.2020200370

13. Dirección de Redes Integradas de Salud Lima Centro - Minsa. Resolución No 129-2019-DMG-
DIRIS-LC [Internet]. [Fecha de acceso: 13 de febrero 2021]. Disponible en: http://www.essalud. gob.pe/hospital-almenara-de-essalud-obtienemaxima-categoria-por-su-alta-especialidad-ycapacidad-resolutiva-en-salud/

14. Xiong $Y$, Sun D, Liu Y, Fan Y, Zhao L, Li X, et al. Clinical and High-Resolution CT Features of the COVID-19 Infection: Comparison of the Initial and Follow-up Changes. Invest Radiol. 2020 Jun; 55(6):332-339. DOI: 10.1097/RLI.00000 00000000674

15. Wang Y, Dong C, Hu Y, Li C, Ren Q, Zhang X, et al. Temporal Changes of CT Findings in $90 \mathrm{~Pa}$ tients with COVID-19 Pneumonia: A Longitudinal Study. Radiology. 2020 Aug; 296(2):E55-E64. DOI: 10.1148/radiol.2020200843

16. Liang $\mathrm{T}$, Liu Z, Wu CC, Jin C, Zhao H, Wang $\mathrm{Y}$, et al. Evolution of CT findings in patients with mild COVID-19 pneumonia. Eur Radiol. 2020 Sep; 30(9):4865-4873. DOI: 10.1007/s00330020-06823-8

17. Pan $Y$, Guan H, Zhou S, Wang Y, Li Q, Zhu T, et al. Initial CT findings and temporal changes in patients with the novel coronavirus pneumonia (2019-nCoV): a study of 63 patients in Wuhan, China. Eur Radiol. 2020 Jun; 30(6):3306-3309. DOI: 10.1007/s00330-020-06731-x

18. Jin YH, Cai L, Cheng ZS, Cheng H, Deng T, Fan $Y P$, et al. A rapid advice guideline for the diagnosis and treatment of 2019 novel coronavirus (2019$\mathrm{nCoV}$ ) infected pneumonia (standard version). Mil Med Res. 2020 Feb 6;7(1):4. DOI: 10.1186/ s40779-020-0233-6

19. Lanza E, Muglia R, Bolengo I, Santonocito OG, Lisi C, Angelotti G, et al. Quantitative chest CT analysis in COVID-19 to predict the need for oxygenation support and intubation. Eur Radiol. 2020 Dec; 30(12):6770-6778. DOI: 10.1007/ s00330-020-07013-2

20. Yuan M, Yin W, Tao Z, Tan W, Hu Y. Association of radiologic findings with mortality of patients infected with 2019 novel coronavirus in Wuhan, China. PLoS One. 2020 Mar 19; 15(3):e0230548. DOI: 10.1371/journal.pone.0230548

21. Long $\mathrm{C}, \mathrm{Xu} \mathrm{H}$, Shen $\mathrm{Q}$, Zhang X, Fan B, Wang C, et al. Diagnosis of the Coronavirus disease (COVID-19): rRT-PCR or CT? Eur J Radiol. 2020 May; 126:108961. DOI: 10.1016/j.ejrad.2020.108961

22. Fang $Y$, Zhang $H$, Xie J, Lin M, Ying L, Pang $P$, et al. Sensitivity of Chest CT for COVID-19: Comparison to RT-PCR. Radiology. 2020 Aug; 296(2):E115E117. DOI: 10.1148/radiol.2020200432

23. Rubin GD, Ryerson CJ, Haramati LB, Sverzellati N, Kanne JP, Raoof S, et al. The Role of Chest Imaging in Patient Management during the COVID-19 Pandemic: A Multinational Consensus Statement from the Fleischner Society. Radiology. $2020 \mathrm{Jul}$; 296(1):172-180. DOI: 10.1148/radiol.2020201365 